\title{
Clinical applications of multimodality cardiac imaging
}

\author{
Alessia Gimelli • Riccardo Liga
}

Received: 16 May 2013/Accepted: 5 September 2013/Published online: 1 October 2013

(C) Italian Association of Nuclear Medicine and Molecular Imaging 2013

\begin{abstract}
Recent decades have brought marked advances in cardiac imaging. Although the single imaging techniques have seen their fields of application expanding, the definition of cardiac pathophysiology frequently demands a multimodality approach. In fact, multimodality imaging offers the chance to perform an integrated anatomo-functional cardiac evaluation in a completely non-invasive manner. This result is obtained by combining the information derived from mainly anatomical imaging modalities, such as multi-slice computed tomography and magnetic resonance, with the functional measures derived from nuclear imaging techniques, single photon emission computed tomography or positron emission tomography. This review will focus on the clinical applications of multimodality cardiac imaging, explaining the need for an integrated evaluation of myocardial structure and function that may enhance the clinician's ability to characterize cardiac pathology.
\end{abstract}

Keywords Multimodality imaging - SPECT ·

MSCT · Myocardial perfusion · Coronary

angiography

\section{Introduction}

In the cardiovascular field, the advances in imaging technologies have been dramatic, and cardiac imaging now

\footnotetext{
A. Gimelli $(\bowtie)$

Fondazione Toscana G. Monasterio, Via Moruzzi, 1, 56100 Pisa,

Italy

e-mail: gimelli@ftgm.it

R. Liga

Scuola Superiore Sant'Anna, Pisa, Italy
}

represents one of the leading causes of the rise in cardiac medical expenditure [1,2].

Notably, the different non-invasive cardiac imaging modalities, single photon emission computed tomography (SPECT), positron emission tomography (PET), multi-slice computed tomography (MSCT), and magnetic resonance (MR), have seen a steady increase in their fields of application together with a considerable enhancement of their diagnostic capabilities.

While giving capital information on regional myocardial blood flow regulation, a standard SPECT myocardial perfusion imaging (MPI) study may also shed light on myocardial function as well as cardiac regional contractility and synchronicity [3, 4]. Likewise, a modern cardiac MSCT evaluation allows physicians to integrate robust evaluation of coronary artery anatomy [5] with non-invasive assessment of myocardial perfusion and contractile function [3, 5].

Nevertheless, even though the abilities of the different techniques have been refined, increasing the types of information that clinicians may acquire from a single study, a multimodality approach is often needed to obtain a comprehensive anatomo-functional cardiac evaluation.

In this context, due to their complementary nature and wide diffusion, nuclear techniques and MSCT have been classically considered ideal partners and constitute the backbone of a modern multimodality cardiac imaging approach [6]. While MSCT can assess the presence of coronary stenoses, nuclear imaging can evaluate their functional consequences, reducing referrals for invasive coronary angiography (ICA) and/or interventions [7] (Figs. 1, 2).

According to this concept, the imaging datasets from the two different modalities performed on separate SPECT or PET devices and MSCT scanners may be co-registered and 


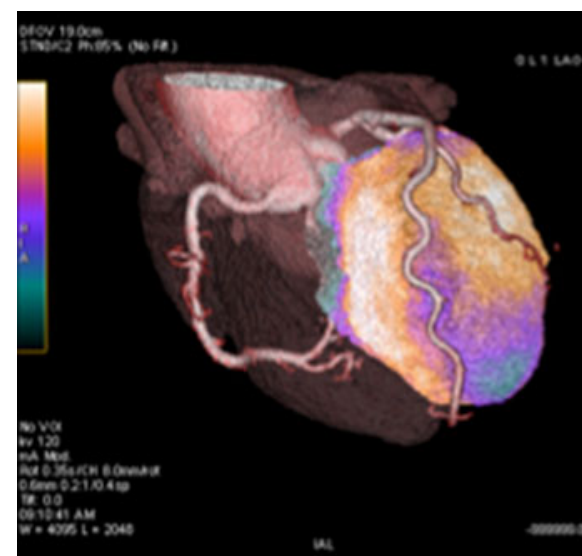

Fig. 1 Hybrid SPECT/MSCT evaluation of a patient with stable angina. A perfusion defect matching with the left-anterior descending coronary artery $(L A D)$ territory is demonstrated. Intravascular
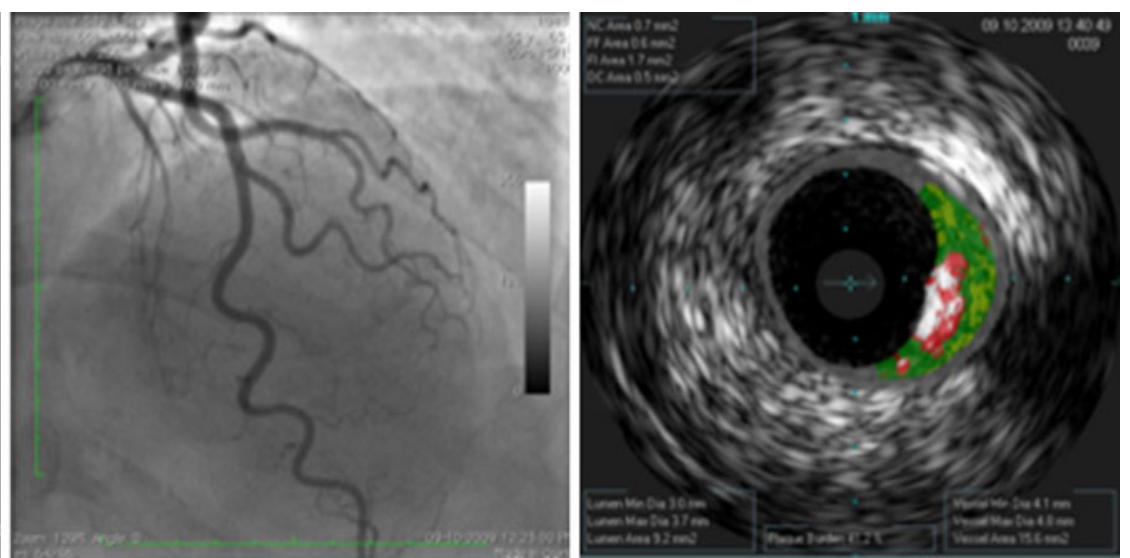

ultrasound evaluation shows the presence of a soft plaque in the mid LAD missed by angiography
Fig. 2 Combined SPECT and MSCT evaluation of a patient with an angiographically significant right coronary artery stenosis and matching perfusion defects

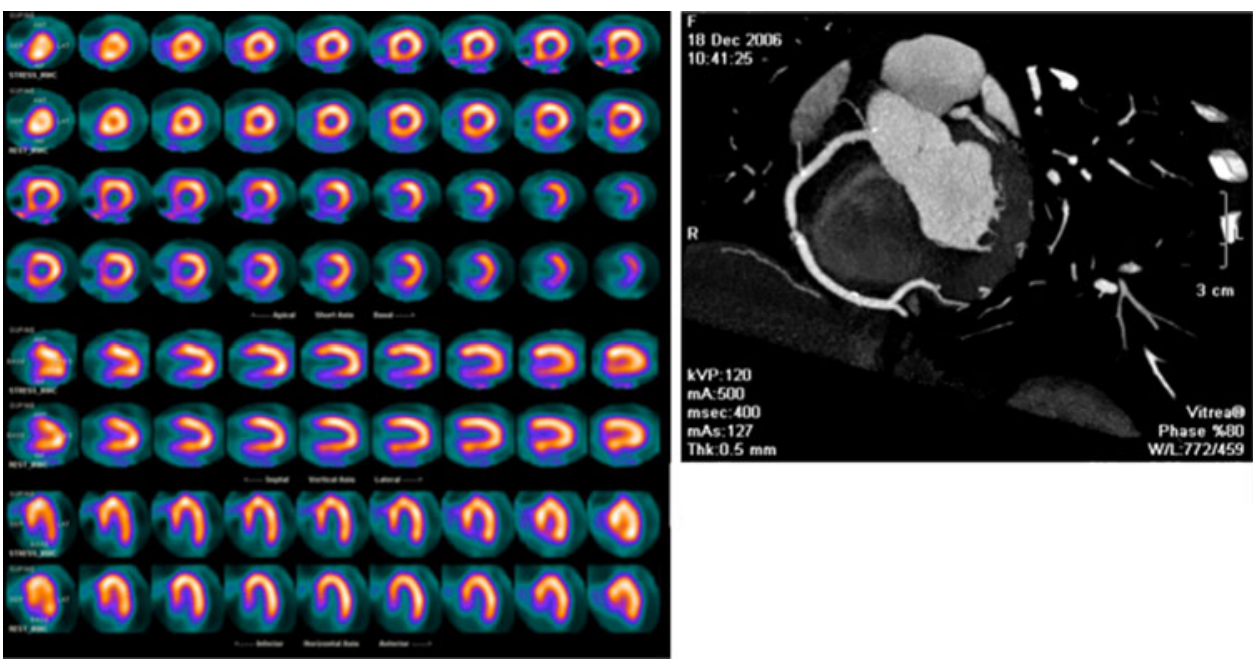

fused by means of dedicated software programs (i.e., software co-registration).

Alternatively, dedicated hybrid scanners that combine either a SPECT or PET device with "state-of-the-art" MSCT may be used (i.e., hardware co-registration), simplifying image fusion while improving patient tolerance.

This review will focus on the clinical applications of multimodality imaging, outlining the major advantages and drawbacks of the single imaging modalities and the reasons for performing an integrated anatomo-functional cardiac evaluation.

\section{Nuclear imaging and coronary CT: two sides of the same coin}

Nuclear imaging techniques are widespread and they are by far the most validated methods for the evaluation of myocardial perfusion [8]. Among the nuclear imaging modalities, cardiac PET has been shown to be more accurate than standard SPECT imaging for the assessment of myocardial perfusion abnormalities [9], providing a unique opportunity to obtain absolute quantification of regional myocardial blood flow [10]. In this regard, a recent meta-analysis showed that PET MPI has high sensitivity $(92.6 \%)$ and specificity $(81.3 \%)$ in identifying significant coronary stenoses [9] (Fig. 3). The reasons for this better accuracy include higher spatial and contrast resolution and depth-independent attenuation correction. In fact, cardiac PET may be able to show subtle alterations of myocardial perfusion and may represent the most costeffective imaging test for the evaluation of myocardial ischemia in patients with intermediate probability of coronary artery disease (CAD) [11].

On the other hand, cardiac SPECT is extremely common and still represents the backbone of nuclear cardiology, serving as a reference technique for the non-invasive evaluation of myocardial perfusion. As classically 


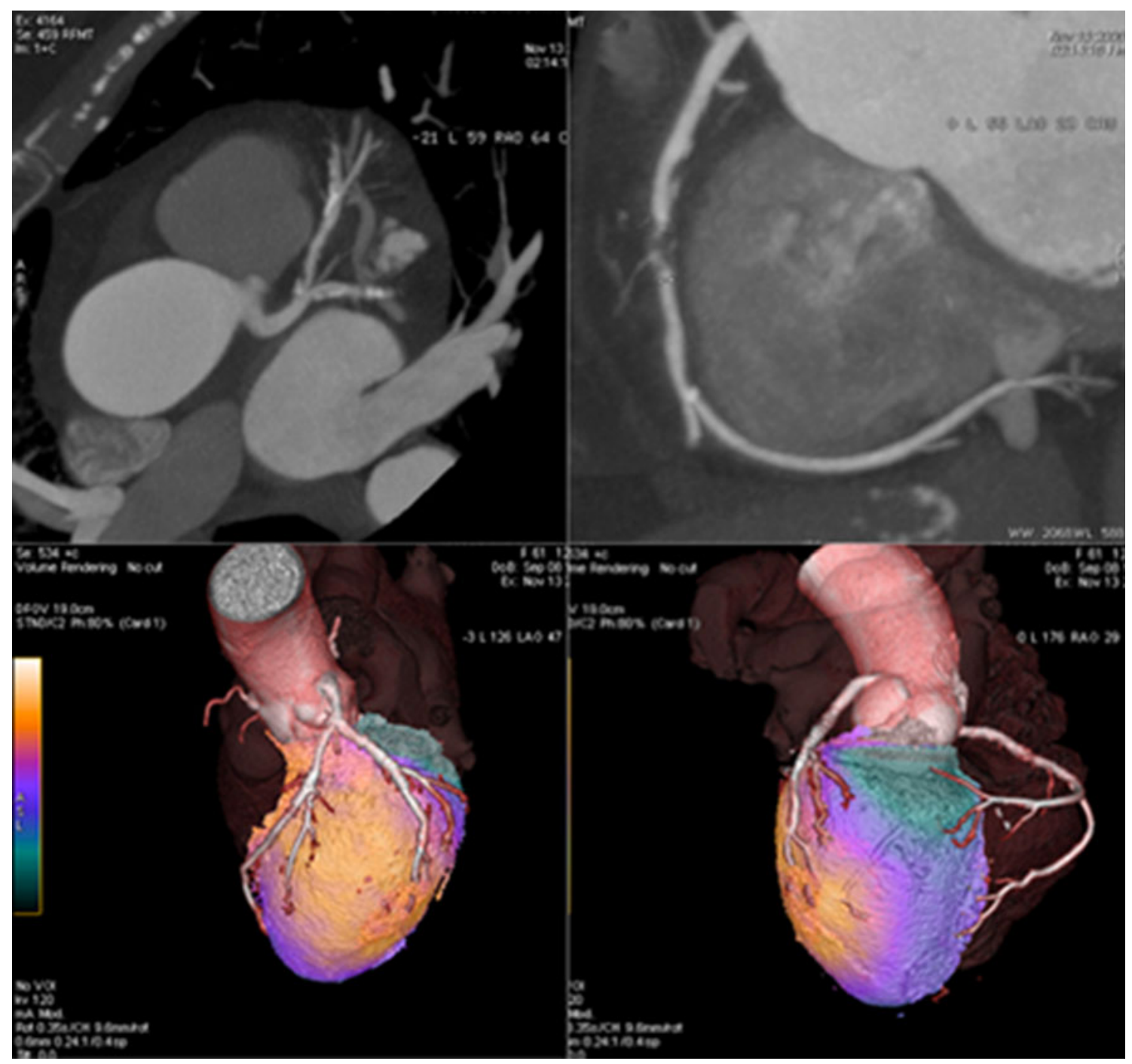

Fig. 3 Hybrid PET and MSCT evaluation of a patient with diffuse calcific coronary artery disease and a large perfusion defect encompassing the right coronary artery territory

reported, the overall sensitivity and specificity of cardiac SPECT for the detection of significant CAD ( $>50 \%$ stenosis) approaches 91 and $87 \%$, respectively [12]. However, while cardiac SPECT MPI represents a cost-effective gatekeeper to ICA in patients with CAD [8], it has several specific drawbacks that should be taken into account. Offering a qualitative evaluation of myocardial perfusion, cardiac SPECT MPI may only unmask coronary territories supplied by the most severe stenosis. Moreover, as shown by Berman et al. [13] a considerable proportion of high-risk patients with significant left main disease may present near-normal MPI scans, possibly as a result of "balanced myocardial ischemia".

The introduction of newer dedicated multi-pinhole solid-state cardiac cameras with cadmium-zinc-telluride (CZT) detectors might overcome these limitations and open up the possibility of obtaining better evaluation of regional myocardial perfusion [14]. As a matter of fact, CZT imaging has been reported to give a lower rate of equivocal results than standard SPECT in the evaluation of myocardial ischemia despite a faster acquisition protocol and considerably lower radiation dose [15].

On the other hand, while nuclear techniques are the reference methods for the evaluation of myocardial perfusion, MSCT is the most widespread and validated method for the non-invasive assessment of coronary anatomy. In patients with a low-to-intermediate prevalence of CAD, 64-multidetector row MSCT angiography showed a sensitivity and specificity to detect $>50 \%$ or $>70 \%$ stenosis of 95 and $83 \%$, respectively, and 94 and $83 \%$, respectively [16].

However, MSCT coronary angiography cannot assess the functional relevance of a given coronary stenosis. Indeed, patients with CAD can show unexpected variability of myocardial perfusion, and nearly half of patients with 
angiographically significant coronary narrowings on MSCT angiography may not have evidence of inducible ischemia in the pertinent vessel territories [17]. Similarly, a sizeable portion of patients with only non-significant coronary stenosis on MSCT may still present inducible ischemia on SPECT [17].

Integrated assessment of coronary anatomy and myocardial perfusion is therefore warranted to reduce inappropriate invasive procedures and improper coronary revascularizations.

\section{Image fusion and hybrid imaging}

Recent studies have suggested that the presence and extent of myocardial ischemia, rather than the angiographic severity of coronary atherosclerosis, might be the major predictor of adverse prognosis in patients with stable CAD [18]. This evidence highlights the importance of an integrated evaluation of coronary anatomy and myocardial perfusion in patients with stable CAD to assess the need for an invasive approach.

Hybrid imaging offers the chance to perform such an evaluation in a completely non-invasive manner, because it combines the high diagnostic accuracy of MSCT coronary angiography with the functional information provided by nuclear imaging in the assessment of regional myocardial perfusion and myocardial viability [8] (Table 1).

To accomplish this objective, two different approaches to obtain image co-registration may be used, i.e., softwareor hardware-based co-registration.

Hardware-based image co-registration relies on the use of hybrid SPECT/CT or PET/CT scanners capable of performing scintigraphic and $\mathrm{CT}$ acquisitions with the patient in a fixed position, allowing better image co-registration. However, due to specific characteristics of cardiac imaging, such as beat-to-beat variation of heart position and respiratory motion, a manual image co-registration is often necessary [19].

Table 1 Advantages of hybrid imaging in patients with CAD

- Higher diagnostic performance compared with single imaging modalities alone

- Adds independent prognostic stratification

- Combines anatomical and functional information obtained with complementary imaging techniques

- Provides anatomical information in patients with global perfusion reduction

- Identification of culprit lesion in multivessel disease

- Combined evaluation of myocardial viability and coronary anatomy to guide coronary revascularization
Alternatively, image datasets may be obtained separately using two distinct scanners and subsequently fused by means of commercially available cardiac fusion software using landmark-based techniques [20]. This approach would exploit the advantage of performing state-of-the-art imaging acquisition with separate MSCT and SPECT scanners, while facilitating imaging workflow.

In this respect, the recent introduction of hybrid scanners equipped with novel dedicated CZT cardiac cameras, characterized by a significantly lower acquisition time than standard cameras, might overcome the unfavorable discrepancy in scan duration between MSCT and nuclear imaging acquisition $[14,15]$.

Another novel field of interest is that of the integration of PET or SPECT devices with high-end MR scanners, offering superior, radiation-free, soft-tissue characterization [21].

\section{Multimodality cardiac imaging: indications and clinical application}

The clinical relevance of hybrid imaging, i.e., the integration of nuclear perfusion techniques and MSCT, has been already documented [22]. In this regard, Gaemperli et al. [20] showed that in almost one-third of the patients submitted to SPECT MPI and MSCT evaluation, the analysis of SPECT/ MSCT fusion images provided added diagnostic information on lesion functional severity over the standard side-by-side analysis. The relative inaccuracy of side-by-side analysis of nuclear imaging data and coronary anatomy may also be related to the high inter-subject morphological variability of the coronary tree. In fact, standard assumptions on vascular territory distribution in MPI are inaccurate in the majority of patients, leading to erroneous allocation of myocardial ischemia to a wrong coronary territory. In this context, the implementation of the hybrid imaging approach, offering the chance to correctly reassign each segment to the pertinent coronary artery, can effectively overcome this limitation, therefore better guiding interventional procedures [23]. In fact, as elegantly shown by Herzog et al. [24] cardiac SPECT/MSCT hybrid imaging allows accurate prediction of ICA findings while minimizing radiation dose and imaging time, thus helping to avoid unnecessary invasive procedures.

Furthermore, the relevant prognostic value of hybrid cardiac SPECT/MSCT imaging has also been reported, and hybrid imaging has been shown to be highly accurate in the overall risk stratification of patients with known or suspected CAD [25].

However, while hybrid imaging seems beneficial in terms of diagnostic accuracy and risk stratification, it obviously exposes the patient to a higher radiation dose. Indeed, because of the need to take into account the 
possible long-term adverse consequences of ionizing radiations, the radiation burden must be continuously monitored and contained. The implementation of up-to-date MSCT acquisition protocols permits a radical reduction (60-80\%) of the radiation dose for an MSCT coronary angiography [8]. Similarly, omission of the rest acquisition when the stress study is normal may significantly reduce the total radiation burden of an MPI protocol without affecting the diagnostic accuracy [26].

Moreover, as hybrid imaging is becoming more widespread in clinical settings, its fields of application are becoming clear. It is generally accepted that, in a low-risk population of patients, cardiovascular imaging should primarily exclude the presence of significant coronary stenoses. In this setting, where MSCT angiography, due to its extremely elevated negative predictive value, might reasonably rule out the presence of CAD in the majority of patients, MPI could be performed only in the case of abnormal or equivocal results, limiting radiation burden and overall costs, as well as referrals for ICA [8].

In a higher risk population, such as older patients with a higher probability of multivessel CAD and presumably more calcified coronary vessels, either SPECT or PET MPI should be considered as a first-line test, while MSCT angiography should be performed in the setting of equivocal MPI findings or in the case of suspected multivessel disease. In these patients, MSCT may be used to evaluate coronary wall anatomy and to quantify coronary artery calcium content, which is strongly associated with myocardial perfusion abnormalities and a validated predictor of adverse prognosis [8].

\section{Multimodality imaging: its role in non-atherosclerotic disease}

Monitoring cardiotoxicity

Cardiotoxicity due to administration of cancer therapeutic agents such as anthracyclines is a well-characterized phenomenon [27]. While guidelines on the screening of chemotherapy-related cardiotoxicity (CRC) are still primarily based on serial assessment of left ventricular (LV) ejection fraction, recent advances in tissue characterization by cardiac MRI might allow early detection of high-risk patients prior to the development of LV dysfunction [28]. On the other hand, cardiac SPECT offers the chance to perform a combined evaluation of myocardial perfusion and function and may be ideal for the early diagnosis of CRC [27].

Considering the strengths and weaknesses of each imaging modality, the future of screening will likely involve an algorithm which will include a multimodality approach to risk stratify patients prior to and shortly after starting chemotherapy, and to allow early diagnosis of initial signs of CRC. However, a comprehensive costeffectiveness evaluation of a multimodality imaging in $\mathrm{CRC}$ is warranted.

\section{Amyloidosis}

The diagnosis of cardiac amyloidosis remains challenging mainly because of the polymorphous clinical presentation. Cardiac MR offers key functional and morphological information on cardiac amyloidosis, providing a unique opportunity to perform non-invasive myocardial tissue characterization [29]. MR is particularly useful in patients with other causes of myocardial wall thickening, easily differentiating cardiac amyloidosis from other causes of myocardial hypertrophy. More recently, the role of nuclear techniques in the characterization of cardiac amyloidosis has been reported. A specific radiotracer, ${ }^{99 \mathrm{~m}} \mathrm{Tc}$-diphosphonate, has been shown to localize cardiac amyloid deposits very sensitively, especially in patients with transthyretin-related disease. Indeed, asymptomatic cardiac transthyretin deposits can be identified through ${ }^{99 \mathrm{~m}} \mathrm{Tc}$-diphosphonate scintigraphy at an early stage when serum cardiac biomarkers, and perhaps even MR, remain non-diagnostic [30]. By contrast, uptake of ${ }^{99 \mathrm{~m}} \mathrm{Tc}$-diphosphonate occurs in about one-third of patients with cardiac light-chain amyloidosis, therefore ${ }^{99 \mathrm{~m}}$ Tc-diphosphonate SPECT can help to distinguish these two types of disease [31].

\section{Advances in nuclear imaging and MSCT: high accuracy at lower radiation dose}

While cardiac SPECT has gained wide application in the clinical setting, its relatively low specificity and high radiation burden represent major drawbacks. Dedicated multipinhole cardiac cameras with CZT detectors have recently been introduced, demonstrating higher spatial resolution than standard SPECT cameras $[14,15]$. In these cameras, the serial alignment of the CZT detectors makes it possible to cover the entire heart at the same time, abolishing the need for camera rotation around the patient. Moreover, the higher photon sensitivity of CZT cameras has allowed a significant reduction of injected dose, significantly reducing radiation burden while preserving diagnostic accuracy [16].

A hybrid imaging approach has been applied to this novel nuclear technology by integrating a CZT camera with a highend 64-row MSCT. The implementation of this novel hybrid technology might allow better resource utilization and a contained radiation dose in patients with CAD [7].

Likewise, the recent introduction of wide-area $>64$-row detector systems has represented a similar revolution in the MSCT field. In fact, thanks to dual-source and novel 

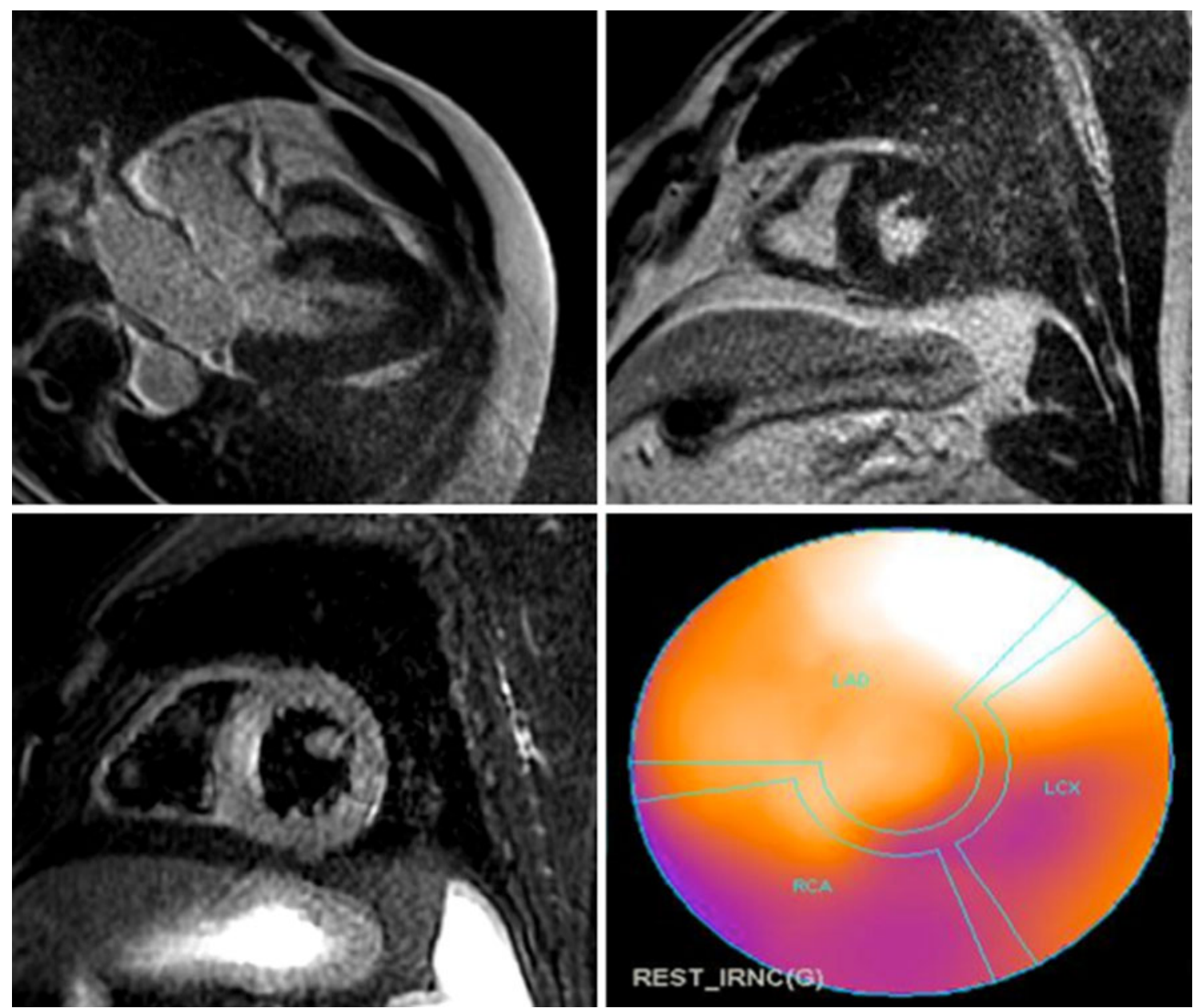

Fig. $4 \mathrm{MR}$ and SPECT assessment of a patient with recent acute myocardial infarction due to mid left-anterior descending coronary artery $(L A D)$ occlusion. While SPECT imaging shows preserved myocardial viability in the LAD territory (lower right panel), MR

320-row detector CT it is possible to obtain whole-heart imaging in one heartbeat, reducing artifacts while allowing a significant dose reduction [7].

Furthermore, the high temporal resolution of these devices allows perfusion imaging of the entire heart at a single moment in time. This is due to greater contrast uniformity during the first pass of contrast, which theoretically enables a combined evaluation of coronary anatomy and myocardial perfusion. Initial validation of this integrated approach against standard SPECT showed a higher level of accuracy than MSCT coronary angiography alone [7].

\section{Hybrid PET/MRI imaging of the heart: present and future}

Hybrid PET/MR tomographs were only recently introduced onto the market due to the significant technical challenges

evaluation, with late gadolinium enhancement (upper panels) and T2weighted short-time inversion recovery sequences (lower left panel), reveals sub-endocardial myocardial necrosis involving the proximal antero-septal segment

inherent in the scanner design. In theory, hybrid PET/MR imaging has the potential to combine the MR-derived superior soft-tissue characterization (Fig. 4) with the extraordinary quality of PET-based functional imaging [21]. However, the additional value that this new modality might bring to the cardiac field has yet to be documented and its potential clinical role defined.

In fact, both MR and PET have independently gained wide acceptance for the assessment of myocardial perfusion and viability in different categories of patients [10, 32]. Thus, by combining the strengths of each modality, hybrid PET/MR imaging might ideally become the future "gold standard" for the evaluation of patients with LV systolic dysfunction, especially in the presence of CAD.

Although PET/MR imaging offers a clear reduction of radiation exposure, by eliminating CT-based tissue characterization, it might consistently increase workflow complexity and operational costs. Ultimately, the great strength 
of PET/MR will lie in its ability to deliver multimodal quantitative imaging parameters based on dynamic data acquisition with both modalities, offering a simultaneous physiological and biological quantitative characterization [33].

While it is still not clear whether PET/MR will be able to offer added value or generate an additional demand for imaging studies that PET/CT cannot satisfy, we expect that the combination of molecular imaging with superb functional characterization of cardiac performance will contribute to better investigation of cardiac pathophysiology and to the development of predictive parameters for tissue recovery and response to therapy.

\section{Conclusions}

The use of multimodality imaging is becoming increasingly widespread in the evaluation of disparate cardiac conditions, allowing, through the 3-dimensional integration of complementary information derived from different imaging techniques, a rapid, non-invasive, anatomo-functional cardiac evaluation.

This approach has been associated with a higher diagnostic accuracy than that of standard single-modality assessment, while containing the overall radiation burden.

Conflict of interest A. Gimelli and R. Liga declare that they have no conflict of interest.

Human and Animal Studies This article does not contain any studies with human or animal subjects performed by any of the authors.

\section{References}

1. Iglehart JK (2006) The new era of medical imaging - progress and pitfalls. N Engl J Med 354:2822-2828

2. Lucignani G (2010) Radiation burden in myocardial imaging: an old concern in the new age of hi-tech, hybrid imaging. Eur J Nucl Med Mol Imaging 37:168-174

3. Schepis T, Gaemperli O, Koepfli P et al (2006) Comparison of 64-Slice CT with gated SPECT for evaluation of left ventricular function. J Nucl Med 47:1288-1294

4. van der Veen BJ, Al Younis I, Ajmone-Marsan N et al (2012) Ventricular dyssynchrony assessed by gated myocardial perfusion SPECT using a geometrical approach: a feasibility study. Eur J Nucl Med Mol Imaging 39:421-429

5. George RT, Arbab-Zadeh A, Miller JM et al (2012) Computed tomography myocardial perfusion imaging with 320-row detector computed tomography accurately detects myocardial ischemia in patients with obstructive coronary artery disease. Circ Cardiovasc Imaging 5:333-340

6. Gaemperli O, Bengel FM, Kaufmann PA (2011) Cardiac hybrid imaging. Eur Heart J 32:2100-2108

7. Fiechter M, Ghadri R, Wolfrum M et al (2012) Downstream resource utilization following hybrid cardiac imaging with an integrated cadmium-zinc-telluride/64-slice CT device. Eur J Nucl Med Mol Imaging 39:430-436

8. Flotats A, Knuuti J, Gutberlet M et al (2011) Hybrid cardiac imaging: SPECT/CT and PET/CT. A joint position statement by the European Association of Nuclear Medicine (EANM), the European Society of Cardiac Radiology (ESCR) and the European Council of Nuclear Cardiology (ECNC). Eur J Nucl Med Mol Imaging 38:201-212

9. Parker MW, Iskandar A, Limone B et al (2012) Diagnostic accuracy of cardiac positron emission tomography versus single photon emission computed tomography for coronary artery disease: a bivariate meta-analysis. Circ Cardiovasc Imaging 5:700-707

10. Bengel FM, Higuchi T, Javadi MS, Lautamäki R (2009) Cardiac positron emission tomography. J Am Coll Cardiol 54:1-15

11. Patterson RE, Eisner RL, Horowitz SF (1995) Comparison of cost-effectiveness and utility of exercise ECG, single photon computed tomography, positron emission tomography, and coronary angiography for diagnosis of coronary artery disease. Circulation 91:54-65

12. Kapur A, Latus KA, Davies G et al (2002) A comparison of three radionuclide myocardial perfusion tracers in clinical practice: the ROBUST study. Eur J Nucl Med Mol Imaging 29:1608-1616

13. Berman DS, Kang X, Slomka PJ et al (2007) Underestimation of extent of ischemia by gated SPECT myocardial perfusion imaging in patients with left main coronary artery disease. J Nucl Cardiol 14:521-528

14. Fiechter M, Ghadri JR, Kuest SM et al (2011) Nuclear myocardial perfusion imaging with a novel cadmium-zinc-telluride detector SPECT/CT device: first validation versus invasive coronary angiography. Eur J Nucl Med Mol Imaging 38:2025-2030

15. Mouden M, Timmer JR, Ottervanger JP et al (2012) Impact of a new ultrafast CZT SPECT camera for myocardial perfusion imaging: fewer equivocal results and lower radiation dose. Eur J Nucl Med Mol Imaging 39:1048-1055

16. Budoff MJ, Dowe D, Jollis JG et al (2008) Diagnostic performance of 64-multidetector row coronary computed tomographic angiography for evaluation of coronary artery stenosis in individuals without known coronary artery disease: results from the prospective multicenter ACCURACY (Assessment by Coronary Computed Tomographic Angiography of Individuals Undergoing Invasive Coronary Angiography) trial. J Am Coll Cardiol 52:1724-1732

17. van Werkhoven JM, Schuijf JD, Gaemperli O et al (2009) Prognostic value of multislice computed tomography and gated single-photon emission computed tomography in patients with suspected coronary artery disease. J Am Coll Cardiol 53:623-632

18. Shaw LJ, Berman DS, Maron DJ et al (2008) Optimal medical therapy with or without percutaneous coronary intervention to reduce ischemic burden: results from the Clinical Outcomes Utilizing Revascularization and Aggressive Drug Evaluation (COURAGE) trial nuclear substudy. Circulation 117:1283-1291

19. Goetze S, Wahl RL (2007) Prevalence of misregistration between SPECT and CT for attenuation-corrected myocardial perfusion SPECT. J Nucl Cardiol 14:200-206

20. Gaemperli O, Schepis T, Valenta I et al (2007) Cardiac image fusion from stand-alone SPECT and CT: clinical experience. J Nucl Med 48:696-703

21. Rischpler C, Nekolla SG, Dregely I, Schwaiger M (2013) Hybrid PET/MR imaging of the heart: potential, initial experiences, and future prospects. J Nucl Med 54:402-415

22. Kajander S, Joutsiniemi E, Saraste M et al (2010) Cardiac positron emission tomography/computed tomography imaging accurately detects anatomically and functionally significant coronary artery disease. Circulation 122:603-613

23. Javadi MS, Lautamäki R, Merrill J et al (2010) Definition of vascular territories on myocardial perfusion images by integration 
with true coronary anatomy: a hybrid PET/CT analysis. J Nucl Med 51:198-203

24. Herzog BA, Husmann L, Buechel RR et al (2011) Rapid cardiac hybrid imaging with minimized radiation dose for accurate noninvasive assessment of ischemic coronary artery disease. Int $\mathbf{J}$ Cardiol 153:10-13

25. Pazhenkottil AP, Nkoulou RN, Ghadri JR et al (2011) Prognostic value of cardiac hybrid imaging integrating single-photon emission computed tomography with coronary computed tomography angiography. Eur Heart J 32:1465-1471

26. Slomka PJ, Dey D, Duvall WL et al (2012) Advances in nuclear cardiac instrumentation with a view towards reduced radiation exposure. Curr Cardiol Rep 14:208-216

27. Schwartz RG, Jain D, Storozynsky E (2013) Traditional and novel methods to assess and prevent chemotherapy-related cardiac dysfunction noninvasively. J Nucl Cardiol 20:443-464

28. Fallah-Rad N, Lytwyn M, Fang T et al (2008) Delayed contrast enhancement cardiac magnetic resonance imaging in trastuzumab induced cardiomyopathy. J Cardiovasc Magn Reson 10:5
29. Karamitsos TD, Piechnik SK, Banypersad SM et al (2013) Noncontrast T1 mapping for the diagnosis of cardiac amyloidosis. JACC Cardiovasc Imaging 6:488-497

30. Minutoli F, Di Bella G, Mazzeo A et al (2013) Comparison between $(99 \mathrm{~m}) \mathrm{Tc}$-diphosphonate imaging and MRI with late gadolinium enhancement in evaluating cardiac involvement in patients with transthyretin familial amyloid polyneuropathy. AJR Am J Roentgenol 200:W256-W265

31. Perugini E, Guidalotti PL, Salvi F et al (2005) Noninvasive etiologic diagnosis of cardiac amyloidosis using 99mTc-3,3-diphosphono-1,2-propanodicarboxylic acid scintigraphy. J Am Coll Cardiol 46:1076-1084

32. Greenwood JP, Maredia N, Younger JF et al (2012) Cardiovascular magnetic resonance and single-photon emission computed tomography for diagnosis of coronary heart disease (CE-MARC): a prospective trial. Lancet 379:453-460

33. Ratib O, Nkoulou R, Schwaiger M (2013) Cardiovascular clinical applications of PET/MRI. Clin Transl Imaging 1:65-71 